\title{
A Study of Pion-Nucleus Interactions in terms of Compound Particles
}

\author{
Tufail Ahmad \\ Applied Science \& Humanities Section, University Polytechnic, Faculty of Engineering \& Technology, \\ Aligarh Muslim University, Aligarh 202 002, India
}

Correspondence should be addressed to Tufail Ahmad; tufailahmadphys@gmail.com

Received 2 October 2013; Accepted 3 November 2013; Published 10 March 2014

Academic Editors: S. Colafranceschi, T.-F. Feng, F.-H. Liu, and I. Lokhtin

Copyright (C) 2014 Tufail Ahmad. This is an open access article distributed under the Creative Commons Attribution License, which permits unrestricted use, distribution, and reproduction in any medium, provided the original work is properly cited.

\begin{abstract}
We report some results on the compound multiplicity distribution at $340 \mathrm{GeV} / \mathrm{c} \pi^{-}$nucleus interactions. Compound multiplicity distribution is found to depend on the target size and the distribution becomes broader. The peak of the distribution shifts towards higher values of the compound particle multiplicity. Mean compound multiplicity is found to vary linearly with grey, heavy, and shower particle multiplicity. Correlations between different particle multiplicities have been studied in detail. Dispersion of compound multiplicity distributions and its ratio with the mean value is observed to obey a linear relationship with different particle multiplicities except for shower particles where dispersion is almost independent of shower particles. Mean normalized multiplicity has also been studied in terms of created charged particles.
\end{abstract}

\section{Introduction}

The investigation of particle-nucleus collisions is fundamental for understanding the nature of the interaction process and these collisions have been studied extensively [1-6]. In experiments on hadron-nucleus (hA) collisions, mostly the attention was paid to the studies of relativistic charged particles produced during these reactions; however, less attempt was made to study the grey and black particles. But the studies on grey particles may provide some useful information because they are envisaged to be produced during or shortly after the passage of leading hadron and hence are expected to remember a part of the history of the reaction. Grey particles may also be taken as good measure of the number of collisions made by the striking hadron inside the target nucleus [7-11]. When a primary particle collides with a target nucleus, the secondary particles produced are shower $\left(N_{s}\right)$, grey $\left(N_{g}\right)$, and black $\left(N_{b}\right)$. These particles are seen in the form of track. The number of grey and black tracks/particles taken together in an event/interaction is termed as heavy track $\left(N_{h}=N_{g}+N_{b}\right)$.

In the study of multiparticle production, multiplicity is one of the most important parameters. This parameter may help in explaining many aspects of particle production process. For the first time Jurak and Linscheid [12] studied some characteristics of proton-nucleus interactions taking shower and grey particles together without making any distinction between them. The number of shower and grey particles taken together in an event was termed as compound particle multiplicity $\left(N_{c}=N_{s}+N_{q}\right)$. Many workers [13-22] analysed the data on nucleus-nucleus (AA) and $\mathrm{hA}$ collisions to investigate some interesting features of compound multiplicity distribution. But less attempt has been made to study the compound multiplicity distribution for pion-nucleus interactions data at $340 \mathrm{GeV} / \mathrm{c}$. Keeping this fact in mind an effort is made in this direction. This will help to compare the particle production process in AA collisions and particle-nucleus collisions.

This paper presents a detailed study of compound multiplicity distribution. In this paper, the phenomenon of particle production in high energy nuclear collisions has been investigated by using the nuclear emulsion technique [23]. Emulsion consists of various elements like hydrogen $(\mathrm{H})$, carbon $(\mathrm{C})$, nitrogen $(\mathrm{N})$, oxygen $(\mathrm{O})$, silver $(\mathrm{Ag})$, and bromine $(\mathrm{Br})$. A thorough study of correlation between different particle multiplicities has been carried out. The variation of mean normalized multiplicity, $R_{A}$, with different types of particles produced is investigated. 


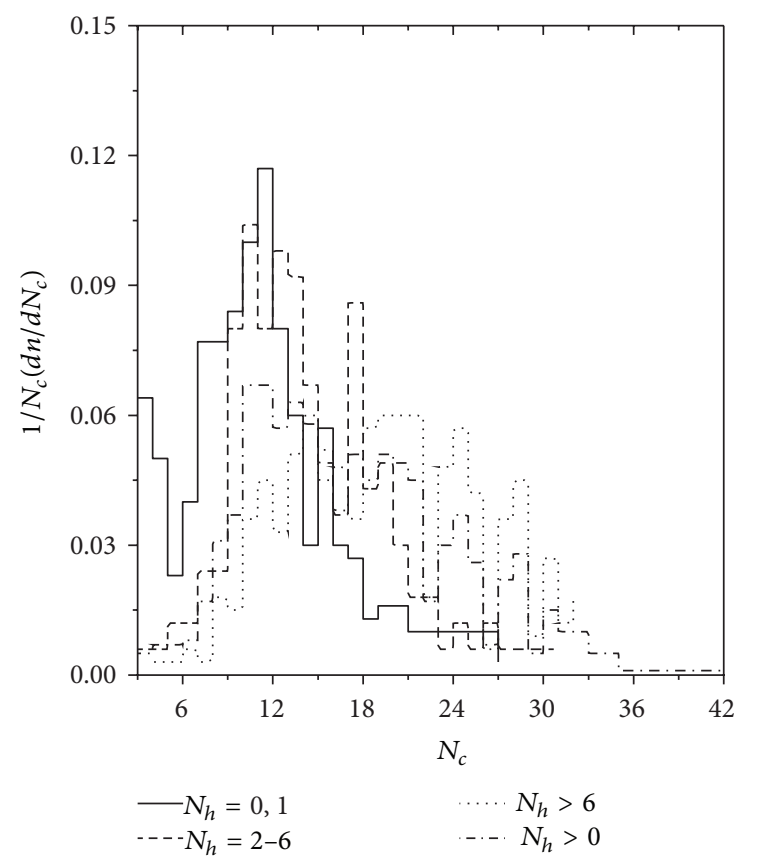

FIGURE 1: Compound multiplicity distribution in different intervals of heavy particle multiplicity.

\section{Experimental Details}

In the present investigation a stack of Ilford-G5 emulsion pellicles each of size $\left(7.50 \times 7.50 \times 0.063 \mathrm{~cm}^{3}\right)$ exposed to a negative pion beam of energy $340 \mathrm{GeV}$ with flux $(0.5-$ 1.5) $\times 10^{4}$ particles $/ \mathrm{cm}^{2}$ at the CERN SPS has been used to collect the data sample. Plates were scanned using M4000 Cooke's series microscopes with $15 \mathrm{x}$ eye pieces and $20 \mathrm{x}$ objectives. The measurement was carried out by using an oilimmersion objective of $100 \mathrm{x}$ magnification. To make sure that data sample does not include any secondary interaction, the primaries of all the events were followed back up to the edge of the plates and only those events whose primary remained parallel to the main direction of the beam and which did not show any significant change in their ionization were finally picked up as genuine primary events.

The secondary tracks emanating from each detected interaction were classified according to emulsion experiment terminology [23] on the basis of their specific ionization $g^{*}\left(=g / g_{o}\right)$, where $g$ is the ionization of the track and $g_{o}$ is the ionization of the primary. The tracks with $g^{*}<1.4$, $1.4 \leq g^{*} \leq 10$, and $g^{*}>10$ were named as shower, grey, and black tracks, respectively. Shower tracks are relativistic charged particles which are mostly produced in the forward hemisphere whereas grey and black tracks are slow particles.

In order to test the target size dependence of compound multiplicity distribution which is shown in Figure 1, four types of targets were used. For $N_{h}=0,1$ nucleon is the target, for $N_{h}>0$, nucleus is the target, for $N_{h}=2-6$, light nuclei (CNO) act as target, and, for $N_{h}>6$, heavy nuclei (AgBr) act as target. The size of the target is decided by the heavy particle multiplicity $N_{h}$.

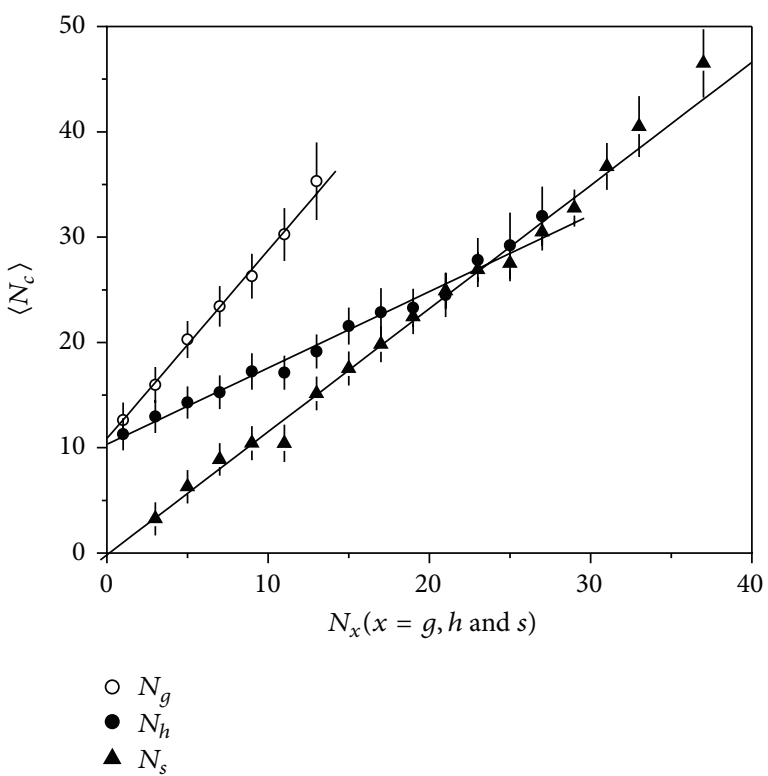

FIGURE 2: Variation of mean compound multiplicity with grey, heavy, and shower particle multiplicity. The $\chi^{2}$ per degrees of freedom $\left(\chi^{2} /\right.$ dof $)$ is $0.09,0.46$, and 0.63 for grey, heavy, and shower particles, respectively.

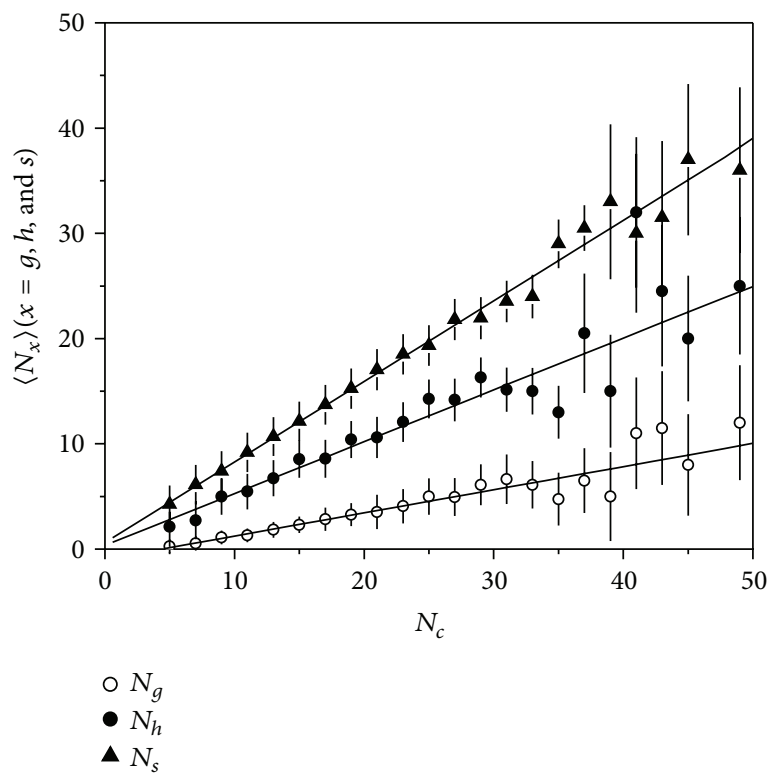

FIGURE 3: Dependence of grey, heavy, and shower particle multiplicity on compound particle multiplicity. The $\chi^{2}$ per degrees of freedom ( $\chi^{2} /$ dof) is $0.90,0.98$, and 0.25 for grey, heavy, and shower particles, respectively.

The interactions, which were produced within $35 \mu \mathrm{m}$ from top or bottom surface of the pellicles and caused by primaries making angles greater than 2 degrees with the mean beam direction, have been excluded from the data. The events were picked up after leaving $3 \mathrm{~mm}$ from the leading edges of the pellicles to avoid any distortion effects. Applying the above rules a sample of 1582 clean events was collected. 


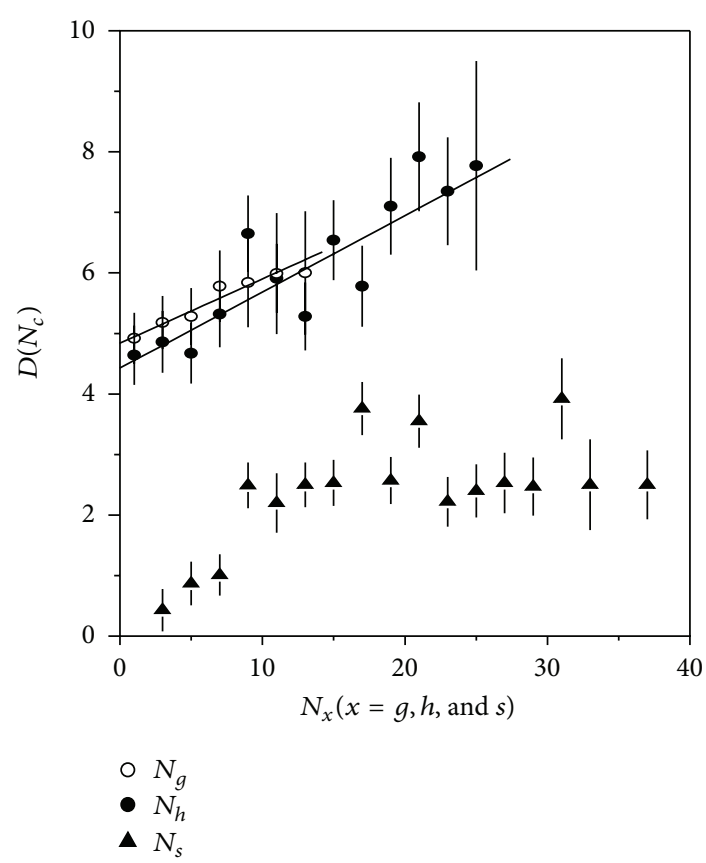

FIGURE 4: Variation of dispersion of compound multiplicity distribution with grey, heavy, and shower particle multiplicity. The $\chi^{2}$ per degrees of freedom $\left(\chi^{2} /\right.$ dof $)$ is 0.75 and 0.96 for grey and heavy particles, respectively.

\section{Experimental Results}

In order to study the target size dependence of compound multiplicity distribution, we have divided the data sample into different $N_{h}$ ensembles. It can be seen from Figure 1 that as target size increases, the compound particle multiplicity distribution becomes broader and broader and the peak of the distribution shifts towards the higher value of $N_{c}$. Other workers $[15,17,18,20]$ have observed similar behaviour. We have tried to study the correlation between different particle multiplicities by using the following relation of the form:

$$
\left\langle N_{i}\left(N_{j}\right)\right\rangle=a_{i j} N_{j}+b_{i j},
$$

where $N_{i}, N_{j}=N_{s}, N_{b}, N_{g}, N_{h}, i \neq j$.

The values of the constants $a_{i j}$ and $b_{i j}$ have been calculated by the method of least squares.

Figure 2 shows the correlations between the $\left\langle N_{c}\right\rangle$ and $N_{g}$, $N_{h}, N_{s}$. It is observed from the figure that $\left\langle N_{c}\right\rangle$ increases linearly with $N_{g}, N_{h}$, and $N_{s}$. The correlations are represented by the following linear relations of the form:

$$
\begin{aligned}
& \left\langle N_{c}\right\rangle=(1.79 \pm 0.21) N_{g}+(10.82 \pm 1.39), \\
& \left\langle N_{c}\right\rangle=(0.72 \pm 0.06) N_{h}+(10.33 \pm 0.89), \\
& \left\langle N_{c}\right\rangle=(1.17 \pm 0.04) N_{s}+(-0.18 \pm 0.89) .
\end{aligned}
$$

Figure 3 shows the variation of $\left\langle N_{g}\right\rangle,\left\langle N_{h}\right\rangle$, and $\left\langle N_{s}\right\rangle$ with $N_{c}$. Here again we find that $\left\langle N_{g}\right\rangle,\left\langle N_{h}\right\rangle$, and $\left\langle N_{s}\right\rangle$ increase

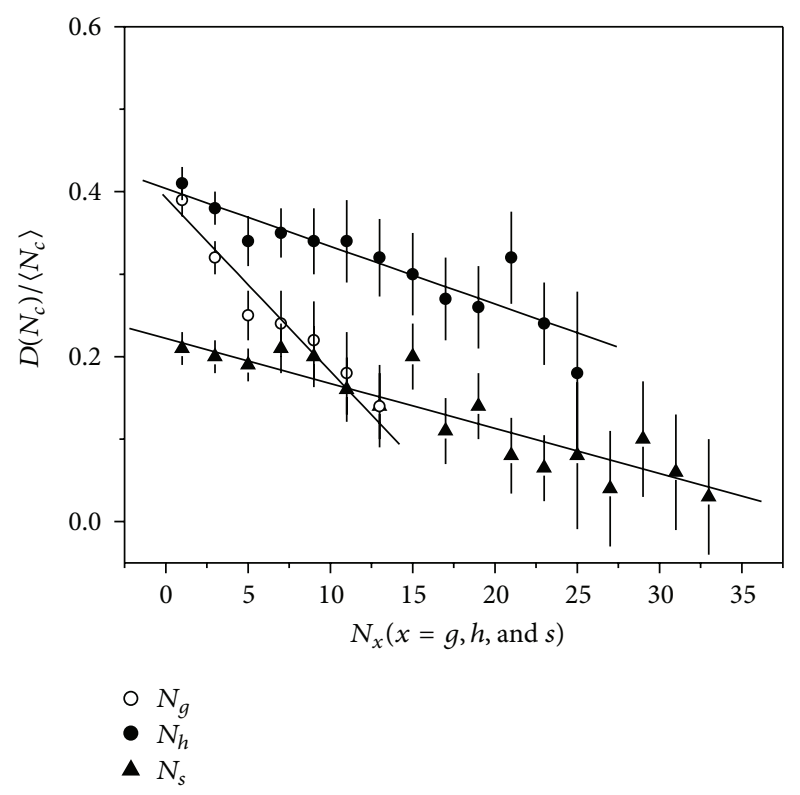

FIGURE 5: Variation of the ratio of dispersion of compound particle multiplicity distribution to mean compound multiplicity with grey, heavy, and shower particle multiplicity. The $\chi^{2}$ per degrees of freedom $\left(\chi^{2} /\right.$ dof $)$ is $0.85,0.63$, and 0.91 for grey, heavy, and shower particles, respectively.

linearly with $N_{c}$. Least square fittings have been made and the following equations are obtained:

$$
\begin{aligned}
\left\langle N_{g}\right\rangle & =(0.21 \pm 0.03) N_{c}+(-0.95 \pm 0.48), \\
\left\langle N_{h}\right\rangle & =(0.49 \pm 0.04) N_{c}+(0.34 \pm 1.04), \\
\left\langle N_{s}\right\rangle & =(0.76 \pm 0.04) N_{c}+(0.63 \pm 1.06) .
\end{aligned}
$$

The variation of the dispersion of compound multiplicity distribution is defined as

$$
D\left(N_{c}\right)=\left(\left\langle N_{c}^{2}\right\rangle-\left\langle N_{c}\right\rangle^{2}\right) .
$$

And the ratio $D\left(N_{c}\right) /\left\langle N_{c}\right\rangle$ with $N_{g}, N_{h}$, and $N_{s}$ are shown in Figures 4 and 5. Almost a linear dependence, which is given by the following equations

$$
\begin{gathered}
D\left(N_{c}\right)=(0.11 \pm 0.06) N_{g}+(4.84 \pm 0.37), \\
D\left(N_{c}\right)=(0.13 \pm 0.03) N_{h}+(4.42 \pm 0.31), \\
\frac{D\left(N_{c}\right)}{\left\langle N_{c}\right\rangle}=(-0.005 \pm 0.00) N_{g}+(0.22 \pm 0.01), \\
\frac{D\left(N_{c}\right)}{\left\langle N_{c}\right\rangle}=(-0.007 \pm 0.00) N_{h}+(0.40 \pm 0.01), \\
\frac{D\left(N_{c}\right)}{\left\langle N_{c}\right\rangle}=(-0.02 \pm 0.00) N_{s}+(0.39 \pm 0.02),
\end{gathered}
$$

is observed in all the cases except in the case of $D\left(N_{c}\right)$ with $N_{s}$ where it is found that dispersion of compound multiplicity distribution is almost independent of charged shower 


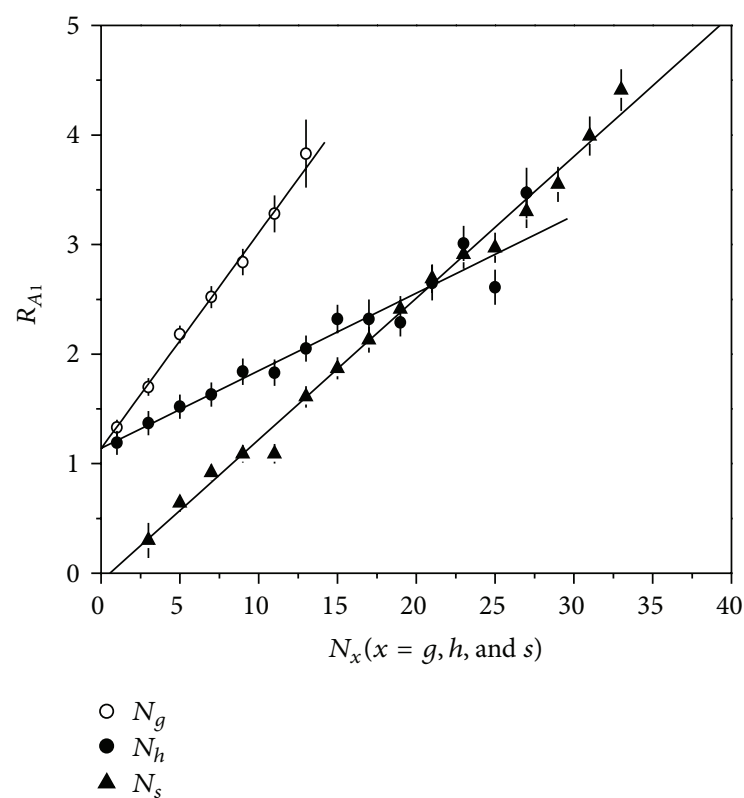

FIGURE 6: Variation of mean normalized multiplicity with grey, heavy, and shower particle multiplicity. The $\chi^{2}$ per degrees of freedom $\left(\chi^{2} / \mathrm{dof}\right)$ is $0.08,0.24$, and 0.84 for grey, heavy, and shower particles, respectively.

particles except at lower values of $N_{s}$, which may be due to statistical fluctuations. This observation agrees qualitatively with collective tube model (CTM) [24-27] prediction.

Another parameter which is used to understand the multiparticle production process is the mean normalized multiplicity, $R_{A}$ (say $R_{A 1}$ ).

Initially this parameter was defined as

$$
R_{A 1}=\frac{\left\langle N_{s}\right\rangle}{\left\langle N_{\mathrm{ch}}\right\rangle}
$$

where $\left\langle N_{s}\right\rangle$ represents the mean number of charged shower particles produced in $\mathrm{hA}$ interactions and $\left\langle N_{\mathrm{ch}}\right\rangle$ is the average number of charged particles in hadron-hadron collisions at almost the same energy. However, later on, some new definitions to the parameter were given in terms of created charged particles. Thus, for example, in $\pi^{-}$-A interactions, this was taken as $[28,29]$

$$
\begin{aligned}
\left(R_{A 2}\right) \pi^{-} A & =\frac{\left\langle N_{s}\right\rangle \mathrm{Cr}}{\left\langle N_{\mathrm{ch}}\right\rangle \mathrm{Cr}} \\
& =\frac{\left(\left\langle N_{s}\right\rangle-0.50\right)}{\left(\left\langle N_{\mathrm{ch}}\right\rangle-1.40\right)} .
\end{aligned}
$$

Here $\mathrm{Cr}$ means created charged particles. For the present investigation, $R_{A 1}$ and $R_{A 2}$ have been calculated by using the following relations:

$$
\begin{gathered}
R_{A 1}=\frac{\left\langle N_{c}\right\rangle}{\left\langle N_{\mathrm{ch}}\right\rangle}, \\
\left(R_{A 2}\right) \pi^{-} A=\frac{\left(\left\langle N_{c}\right\rangle-0.50\right)}{\left(\left\langle N_{\mathrm{ch}}\right\rangle-1.40\right)} .
\end{gathered}
$$

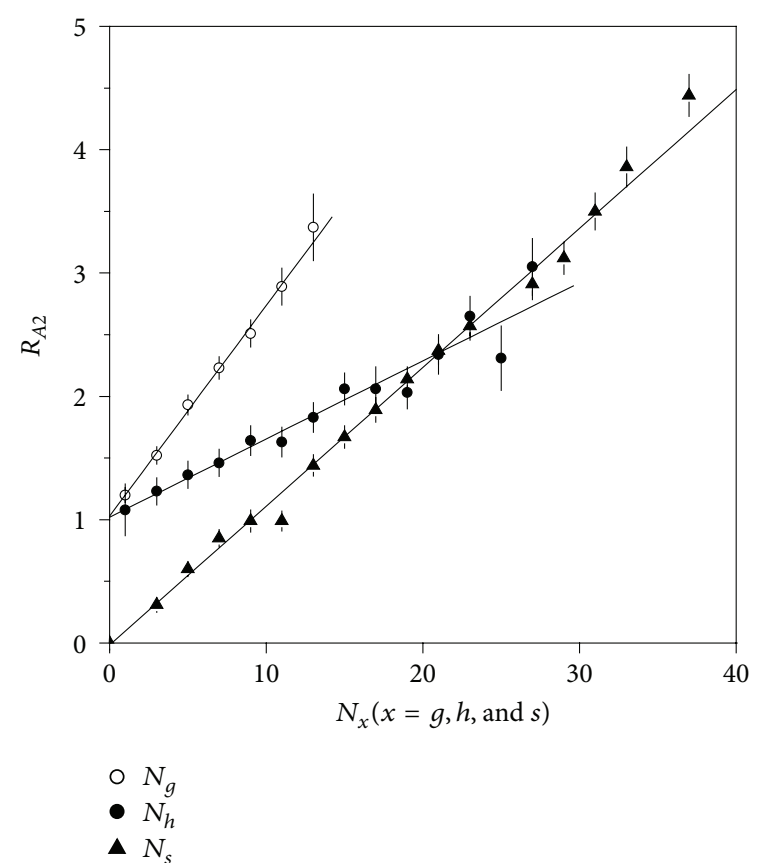

FIGURE 7: Variation of mean normalized multiplicity in terms of created charged particles with grey, heavy, and shower particle multiplicity. The $\chi^{2}$ per degrees of freedom $\left(\chi^{2} /\right.$ dof $)$ is $0.09,0.26$, and 0.71 for grey, heavy, and shower particles, respectively.

The variations of $R_{A 1}$ and $R_{A 2}$ with $N_{g}, N_{h}$, and $N_{s}$ are shown in Figures 6 and 7. One may notice from the figures that $R_{A 1}$ and $R_{A 2}$ depend more strongly on $N_{g}$ than $N_{h}$ or $N_{s}$ and the data is best fitted by the following lines:

$$
\begin{aligned}
& R_{A 1}=(0.20 \pm 0.01) N_{g}+(1.14 \pm 0.06), \\
& R_{A 1}=(0.07 \pm 0.00) N_{h}+(1.14 \pm 0.06), \\
& R_{A 1}=(0.12 \pm 0.00) N_{s}+(-0.07 \pm 0.05), \\
& R_{A 2}=(0.17 \pm 0.01) N_{g}+(1.03 \pm 0.05), \\
& R_{A 2}=(0.06 \pm 0.00) N_{h}+(1.02 \pm 0.07), \\
& R_{A 2}=(0.11 \pm 0.00) N_{s}+(-0.01 \pm 0.03)
\end{aligned}
$$

The diffractive excitation model $[30,31]$ and energy flux cascade (EFC) model [32] predict that $R_{A}$ should vary with the number of collisions made by the impinging hadron inside the struck nucleus, $v$, as $R_{\mathrm{A}}=a+b v, a=b=1 / 2$ (in the diffractive excitation model) and $a=2 / 3$ and $b=1 / 3$ (in the EFC). The CTM [24-27] predicts that $R_{A}$ should vary as $\boldsymbol{\nu}^{1 / 4}$, since the number of grey particles, $N_{g}$, is considered as the best measure of the number of collisions. Thus, none of these predictions of models agree with experimental results.

\section{Concluding Remarks}

On the basis of the results presented we may conclude the following. 
(i) The compound multiplicity distribution is found to become broader as the target size increases so one can say that it is target size dependent.

(ii) Mean compound multiplicity is found to vary linearly with grey, heavy, and shower particle multiplicity.

(iii) The variation of the ratio of the dispersion of compound particle multiplicity distribution to the mean compound multiplicity with shower particle multiplicity supports collective tube model.

(iv) The dependence of mean normalized multiplicity and mean normalized multiplicity in terms of created charged particles on grey particle multiplicity is against the collective tube model prediction.

\section{Conflict of Interests}

The author declares that there is no conflict of interests regarding the publication of this paper.

\section{References}

[1] Z. V. Anzon, I. Y. Chasnikov, A. S. Gaitinov et al., "A study of inelastic pion-nucleus interactions at $200 \mathrm{GeV} / \mathrm{c}$ in an emulsion," Nuclear Physics B, vol. 129, no. 2, pp. 205-234, 1977.

[2] I. Otterlund, E. Stenlund, B. Andersson et al., "Nuclear interactions of $400 \mathrm{GeV}$ protons in emulsion," Nuclear Physics B, vol. 142, no. 4, pp. 445-462, 1978.

[3] M. El-Nadi, O. E. Badawy, M. K. Hegab et al., "Inelastic interactions of $340 \mathrm{GeV} / \mathrm{c}$ - With emulsion nuclei," Physical Review D, vol. 27, no. 1, pp. 12-18, 1983.

[4] R. K. Shivpuri and A. Kotha, "Multiplicity, rapidity, and rapidity correlations in $800 \mathrm{GeV}$ proton-nucleus interactions," Physical Review D, vol. 35, no. 11, pp. 3508-3511, 1987.

[5] A. Tufail, S. Ahmad, A. R. Khan, M. Zafar, and M. Shafi, "Nuclear interactions of $340 \mathrm{GeV}$ pions in emulsion," Physical Review D, vol. 42, no. 7, pp. 2187-2193, 1990.

[6] A. Tufail, S. Ahmad, and M. Zafar, "Features of forwardbackward multiplicity correlations in hadronic collisions," Canadian Journal of Physics, vol. 74, no. 3-4, pp. 141-144, 1996.

[7] B. Andersson, I. Otterlund, and E. Stenlund, "On the correlation between fast target protons and the number of hadron-nucleon collisions in high-energy hadron-nucleus reactions," Physics Letters B, vol. 73, no. 3, pp. 343-346, 1978.

[8] M. K. Hegab and J. Hüfner, "How often does a high-energy hadron collide inside a nucleus?" Physics Letters B, vol. 105, no. 2-3, pp. 103-106, 1981.

[9] M. K. Hegab and J. Hüfner, "The distribution of grey particles in very high-energy hadron-nucleus collisions," Nuclear Physics A, vol. 384, no. 3, pp. 353-370, 1982.

[10] W. Q. Chao, M. K. Hegab, and J. Hüfner, "Multiparticle production in hadron-nucleus collisions around $100 \mathrm{GeV}$," Nuclear Physics A, vol. 395, no. 2, pp. 482-508, 1983.

[11] P. L. Jain, K. Sengupta, and G. Singh, "Multiplicity dependence on the number of projectile collisions in hadron-nucleus interactions," Il Nuovo Cimento A, vol. 99, no. 1, pp. 9-20, 1988.

[12] A. Jurak and A. Linscheid, "Some characteristics of compound shower and grey tracks multiplicity distributions produced in proton emulsion interactions," Acta Physica Polonica B, vol. 8, pp. 875-886, 1977.

[13] D. Ghosh, A. Mukhopadhyay, A. Ghosh, R. Sengupta, and J. Roy, "Multiplicity characteristics of heavy-ion interactions at $4.5 \mathrm{GeV} / \mathrm{yc}$ per nucleon,” Nuclear Physics A, vol. 499, no. 4, pp. $850-860,1989$.

[14] T. Ahmad and M. Irfan, "Features of compound multiplicity in heavy-ion interactions at $4.5 \mathrm{~A} \mathrm{GeV} / \mathrm{c}$," Physical Review $C$, vol. 44, no. 4, pp. 1555-1558, 1991.

[15] M. S. Khan, S. S. Ali, P. Singh et al., "Some interesting results on compound multiplicity in ${ }^{12} \mathrm{C}$-nucleus reactions at $4.5 \mathrm{~A}$ GeV/c," Canadian Journal of Physics, vol. 75, no. 8, pp. 549-557, 1997.

[16] N. N. Abd-Allah and M. Mohery, "Features of the compound multiplicity of the interactions of ${ }^{24} \mathrm{Mg}$ and ${ }^{82} \mathrm{Si}$ ions with emulsion nuclei at $4.5 \mathrm{~A} \mathrm{GeV/c,"} \mathrm{Turkish} \mathrm{Journal} \mathrm{of} \mathrm{Physics,} \mathrm{vol.}$ 25, no. 2, pp. 109-119, 2001.

[17] Z. Abou-Moussa, "Compound multiplicity in the collisions of $4.1 \mathrm{~A} \mathrm{GeV} / \mathrm{c}^{22} \mathrm{Ne}$ nuclei with nuclear emulsion," Canadian Journal of Physics, vol. 80, no. 3, pp. 109-117, 2002.

[18] D.-H. Zhang, C.-L. He, Y. Yuan et al., "Features of compound multiplicity in ${ }^{16} \mathrm{O}-\mathrm{Em}$ interactions at $4.5 \mathrm{~A} \mathrm{GeV} / \mathrm{c}$," Chinese Journal of Physics, vol. 44, no. 6, pp. 405-417, 2006.

[19] M. Mohery and M. Arafa, "Some characteristics of the compound multiplicity in high-energy nucleusnucleus interactions," International Journal of Modern Physics E, vol. 20, no. 8, pp. 1735-1754, 2011.

[20] C.-Y. Bai and D.-H. Zhang, "Study of compound particle production in ${ }^{84} \mathrm{Kr}$-emulsion interactions at $1.7 \mathrm{~A} \mathrm{GeV,"} \mathrm{Chinese}$ Physics C, vol. 35, no. 4, pp. 349-354, 2011.

[21] D. Ghosh, J. Roy, K. Sengupta, M. Basu, S. Naha, and A. Bhattacharya, "A new approach for the study of hadron-nucleus interactions through compound multiplicity," Hadronic Journal, vol. 5, pp. 163-173, 1981.

[22] T. Ahmad, N. Ahmad, S. Ahmad, and M. Zafar, "Characteristics of compound multiplicity in hadron-nucleus collisions," Indian Journal of Pure and Applied Physics, vol. 48, no. 12, pp. 855-859, 2010.

[23] W. H. Barkas, Nuclear Research Emulsions, Academic Press, New York, NY, USA, 1963.

[24] F. Takagi, "A model for mean multiplicity in hadron-nucleus collisions," Lettere al Nuovo Cimento, vol. 14, no. 15, pp. 559-564, 1975.

[25] F. Takagi, "Universality of mean charged hadron multiplicities in particle and nuclear collisions," Progress Theoretical Physics, vol. 57, no. 3, pp. 939-952, 1977.

[26] G. Berlad, A. Dar, and G. Eilam, "Multiparticle production in particle-nucleus collisions at high energies," Physical Review D, vol. 13, no. 1, pp. 161-165, 1976.

[27] M. Ta-Chung, "Multiparticle production in high-energy hadron-nucleus collisions," Physical Review D, vol. 15, no. 1, pp. 197-205, 1977.

[28] T. Aziz, M. Zafar, M. Irfan, A. Ahmad, and M. Shafi, "Analysis of multiparticle production data on proton-nucleus collisions using a new variable," Pramana, vol. 11, no. 3, pp. 323-332, 1978.

[29] T. Aziz and M. Zafar, "A model for multiparticle production in high energy hadronic collisions," Pramana, vol. 13, no. 1, pp. 81$88,1979$. 
[30] A. Dar and J. Vary, "Method to distinguish between multiparticle production mechanisms," Physical Review D, vol. 6, no. 9, pp. 2412-2417, 1972.

[31] P. M. Fishbane and J. S. Trefil, "Hadronic scaling in the scattering of composite systems," Physical Review D, vol. 8, no. 5, pp. 1467-1485, 1973.

[32] K. Gottfried, "Space-time structure of hadronic collisions and nuclear multiple production," Physical Review Letters, vol. 32, no. 17, pp. 957-961, 1974. 

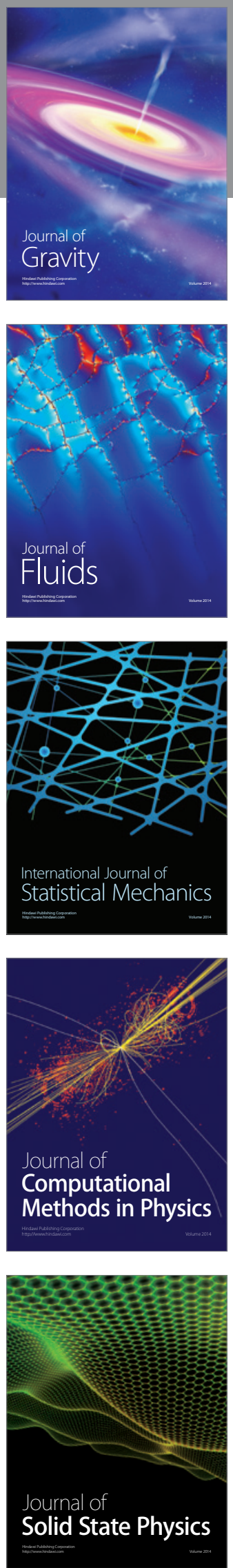

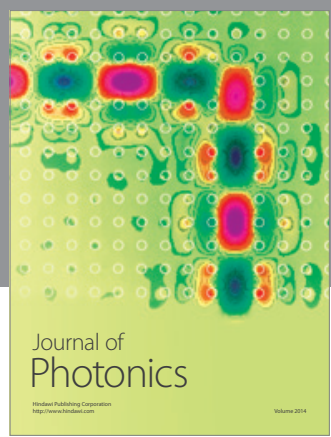

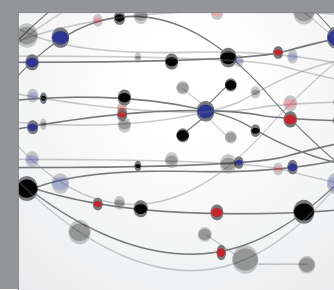

The Scientific World Journal

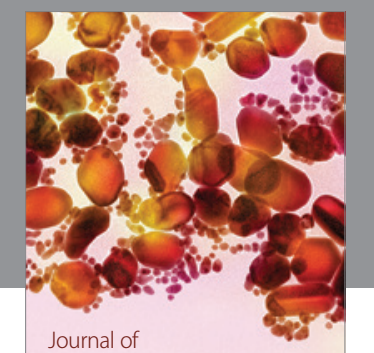

Soft Matter
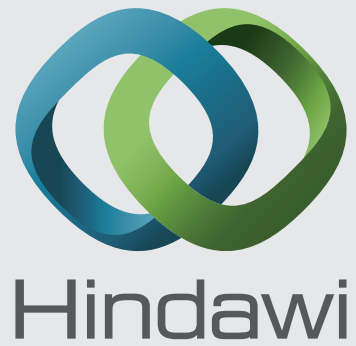

Submit your manuscripts at

http://www.hindawi.com
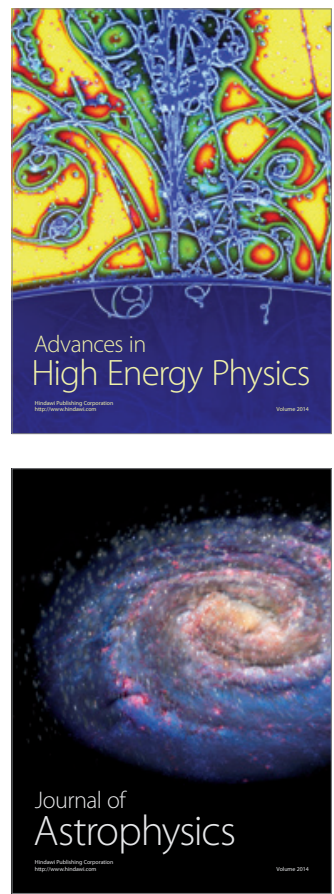
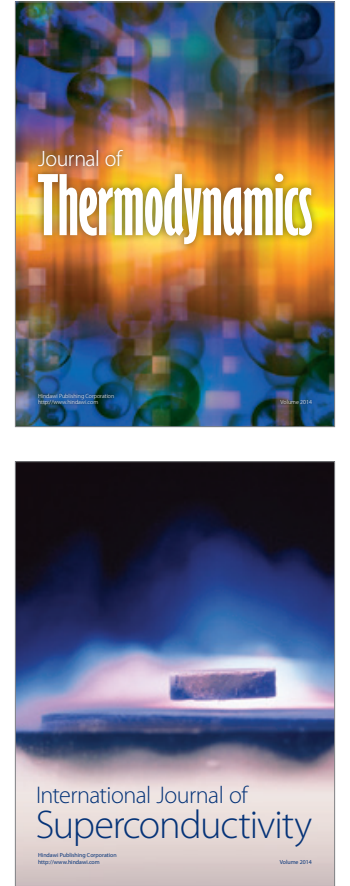
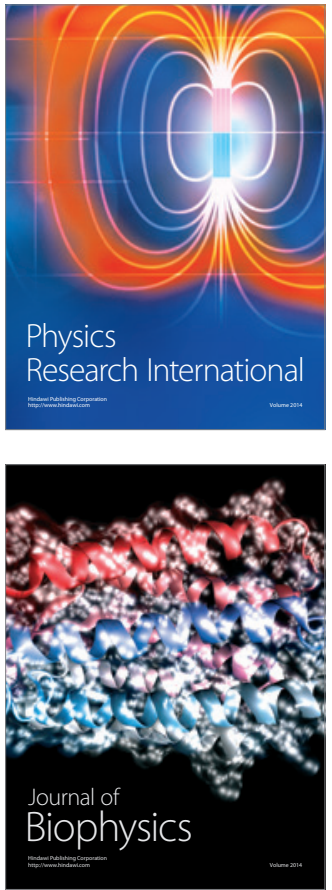
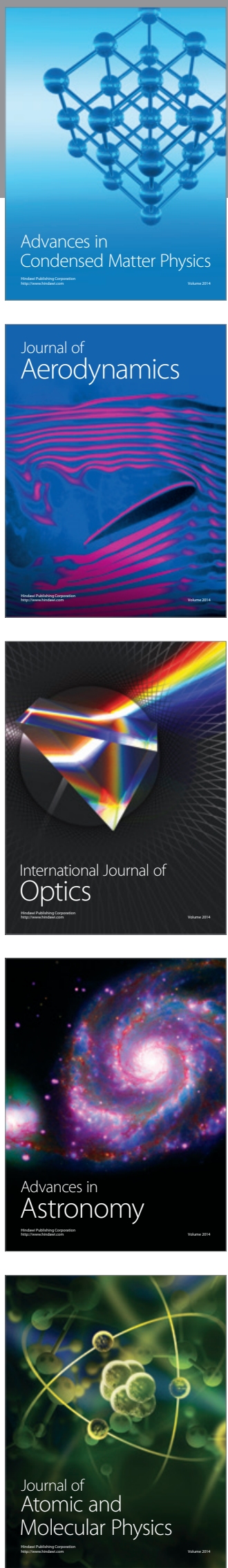